\title{
Measuring robot kinematics description and its workspace
}

\author{
Zdeněk Úředníček ${ }^{1}$ and, Rudolf Drga ${ }^{2}$ \\ ${ }^{1}$ UTB FAl ve Zlíně, Department of Automation and Control Engineering, Nad Stráněmi 451176005 Zlín, Czech Republic, \\ ${ }^{2}$ UTB FAl ve Zlíně, Department of Security Engineering ,Nad Stráněmi 451176005 Zlín, Czech Republic,
}

\begin{abstract}
Text deals with 6 degree of freedom measuring robot kinematics description enabling manipulation with measuring effector in 3D space. Mentioned description flowing from Denavit- Hartenberg notation for serial ordering of ideally stiff bodies connected by kinematic pairs, makes possible to create complete set of transformation matrices for coordinates transformation from distal to the adjoining proximal Cartesian coordinate system, which enables to calculate the coordinates of arbitrary structure point by means of joints' coordinates. Thenceforth it makes possible to determine standby and available robot workplace and mainly to perform the analysis of one fundamental control system problem of such robots- i. e. solving inverse kinematic problem.
\end{abstract}

\section{Introduction}

On solving of problem deal with parameters collection in defined 3D space points, or with theirs influence, there're not only in laboratory conditions, but also in real practice exploited robotic systems enabling to smartly adjust required measuring or active effector position.

Knowledge in kinematic relationships among required effector position and robotic system joints' coordinates enables to determine both basic robot workspaces, which is important in light of measuring robotic system proposal. A primarily, it makes possible to define, to analyse and eventually to solve so - called inverse kinematic problem. I.e. determination robot joint coordinates for effector required position in $3 \mathrm{D}$ space.

\section{Kinematic structure make-up}

Figure 1 shows described measuring robot real kinematic structure make-up. This make-up is created that way, that it isn't possible to divide formulated problem solving to manipulator problem ensuring so-called wrist point position in 3D space and standard wrist created by three rotary kinematic pairs with orthogonal axis decussate in one point - wrist point.

Two rotary joints are orthogonal and intersect always in one point, third rotary joint axis generally intersects only one axis from previous and not orthogonally yet.

As a consequence is further mentioned direct kinematic problem description (algebraic relation among joint coordinates and point effector Cartesian coordinates), which is solvable (if ever) only numerically. It complicates for example control system operation in case of need on-line generation of requested values of joint coordinates at autonomous robot behaviour.

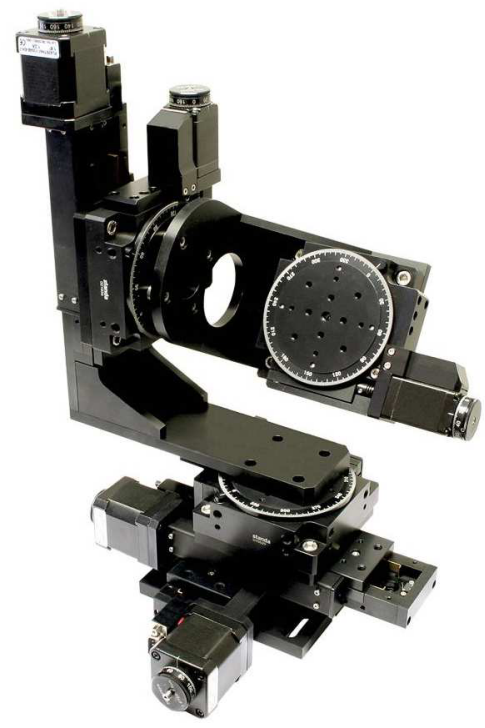

Figure 1 Real measuring robot mechanical structure.

\subsection{Co-ordinate systems according to Denavit- Hartenberg}

Figure 2 shows select ordering of Cartesian co- ordinate systems on described kinematic structure and basic dimensions and Table 1. shows then associated Denavit Hartenberg parameters (DH ).

Corresponding author: urednicek@fai.utb.cz 


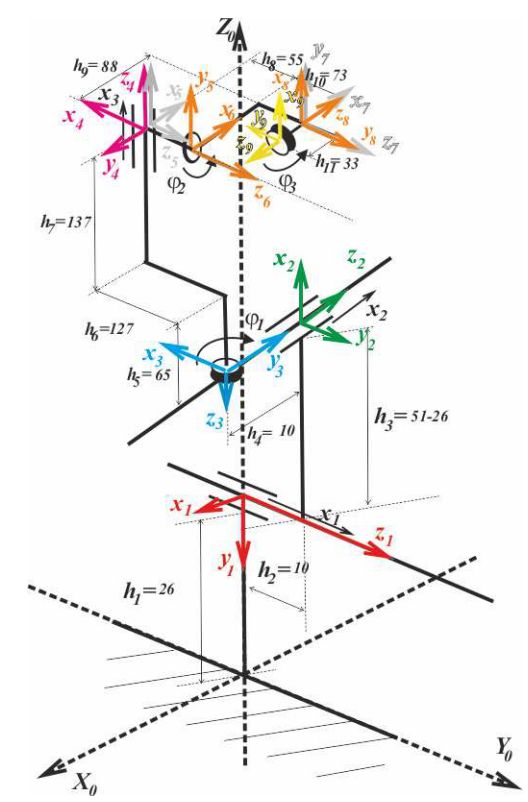

Figure 2 Measuring robot DH co-ordinate systems introduction.

Table 1 DH parameters of axis systems.

\begin{tabular}{|c|c|c|c|c|}
\hline parametr & $a_{i}$ & $\alpha_{i}$ & $d_{i}$ & $\Theta_{\mathbf{i}}$ \\
\hline 1 & $z_{0} \rightarrow z_{1}$ along $x_{1}=0$ & $z_{0} \rightarrow z_{1}$ around $x_{1}=-90^{\circ}$ & $x_{0} \rightarrow x_{1}$ along $z_{0}=h_{1}$ & $\mathbf{x}_{0} \rightarrow \mathbf{x}_{1}$ around $\mathrm{z}_{0}=0^{0}$ \\
\hline 2 & $\mathrm{z}_{1} \rightarrow \mathrm{I}_{2}$ along $\mathrm{X}_{2}=\mathbf{h}_{3}$ & $\mathbf{z}_{1} \rightarrow \mathrm{z}_{2}$ around $\mathbf{x}_{2}=90^{\circ}$ & $x_{1} \rightarrow x_{2}$ along $z_{1} \Rightarrow h_{2}+x_{1}$ & $\mathrm{I}_{1} \rightarrow \mathrm{I}_{2}$ around $\mathrm{Z}_{1}=-90^{\circ}$ \\
\hline 3 & $z_{2} \rightarrow z_{3}$ along $x_{3}=0$ & $z_{2} \rightarrow z_{3}$ around $0 x_{3}=90^{\circ}$ & $\mathbf{x}_{2} \rightarrow \mathbf{x}_{3}$ along $\mathbf{z}_{2}=-\mathbf{h}_{4}-\mathbf{X}_{2}$ & $\mathrm{X}_{2} \rightarrow \mathrm{X}_{3}$ around $\mathrm{Z}_{2}=90^{\circ}$ \\
\hline 4 & $\mathrm{z}_{3} \rightarrow \mathrm{z}_{4}$ along $\mathrm{x}_{4}=\mathbf{h}_{6}$ & $z_{3} \rightarrow Z_{4}$ around $x_{4}=180^{\circ}$ & 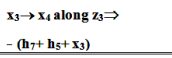 & $\mathrm{x}_{3} \rightarrow \mathrm{x}_{4}$ around $\mathrm{r}_{3}=\varphi_{1}$ \\
\hline 5 & $\mathrm{z}_{\mathrm{t} \rightarrow \mathrm{z}_{\mathrm{s}} \text { along } \mathrm{x}_{\mathrm{s}}=\mathbf{0}}$ & $\mathrm{z}_{4} \rightarrow \mathrm{z}_{5}$ around $\mathrm{x}_{5}=90^{\circ}$ & $x_{4} \rightarrow x_{5}$ along $z_{4}=0$ & $x_{4} \rightarrow x_{5}$ around $z_{4}=-90^{\circ}$ \\
\hline 6 & $z_{5} \rightarrow z_{6}$ along $x_{6}=0$ & $z_{5} \rightarrow z_{6}$ around $x_{6}=0^{0}$ & $\mathrm{x}_{5} \rightarrow \mathrm{X}_{6}$ along $\mathrm{z}_{3} \rightarrow \mathrm{h}_{8}$ & $\mathrm{x}_{\mathrm{s}} \rightarrow \mathrm{x}_{6}$ around $\mathrm{z}_{5}=0^{0}$ \\
\hline 7 & $\mathbf{z}_{6} \rightarrow \mathbf{z}_{7}$ along $\mathbf{z}_{7}=\mathbf{h}$ & $z_{6} \rightarrow z_{7}$ around $x_{7}=0^{0}$ & $\mathbf{x}_{6} \rightarrow \mathbf{x}_{7}$ along $\mathrm{z}_{6} \rightarrow \mathbf{h}_{10}$ & $\mathbf{x}_{6} \rightarrow \mathrm{x}_{7}$ around $\mathrm{z}_{6}=\varphi_{2}$ \\
\hline 8 & $z_{7 \rightarrow} \rightarrow z_{8}$ along $1 x_{8}=0$ & $\mathrm{Z} \rightarrow \rightarrow \mathrm{Z}_{8}$ around $\mathrm{x}_{8}=90^{\circ}$ & $\mathbf{x}_{7} \rightarrow \mathbf{x}_{8}$ along $\mathrm{z}_{7} \rightarrow 0$ & $\mathrm{X}_{7} \rightarrow \mathrm{X}_{8}$ around $\mathrm{Z}_{7}=90^{\circ}$ \\
\hline 9 & $z_{s} \rightarrow z_{9}$ along $x_{9}=0$ & $z_{8} \rightarrow z_{9}$ around $x_{9}=180^{\circ}$ & $\mathbf{x}_{8 \rightarrow \mathbf{x}} \rightarrow \mathbf{x}_{9}$ along $\mathrm{z}_{8} \rightarrow-\mathbf{h}_{11}$ & $\mathbf{I}_{8} \rightarrow \mathbf{I}_{9}$ around $\mathrm{Z}_{\mathrm{S}}=-\boldsymbol{\varphi}_{3}$ \\
\hline
\end{tabular}

\subsection{Homogenous transformation matrices}

Because for transformation homogenous matrix for coordinates of proximal and distal neighbouring systems reads

$$
{ }^{i-1} \mathrm{~T}_{i}=\left[\begin{array}{cccc}
\cos \theta_{i} & -\cos \alpha_{i} \sin \theta_{i} & \sin \alpha_{i} \sin \theta_{i} & a_{i} \cos \theta_{i} \\
\sin \theta_{i} & \cos \alpha_{i} \cos \theta_{i} & -\sin \alpha_{i} \cos \theta_{i} & a_{i} \sin \theta_{i} \\
0 & \sin \alpha_{i} & \cos \alpha_{i} & d_{i} \\
0 & 0 & 0 & 1
\end{array}\right]
$$

it is possible to write for homogenous transformation matrices among particular coordinate systems from Figure 2

$$
\begin{gathered}
{ }^{0} \mathrm{~T}_{1}=\left[\begin{array}{cccc}
1 & 0 & 0 & 0 \\
0 & 0 & 1 & 0 \\
0 & -1 & 0 & h_{1} \\
0 & 0 & 0 & 1
\end{array}\right] ; \quad{ }^{1} \mathrm{~T}_{2}=\left[\begin{array}{cccc}
0 & 0 & -1 & 0 \\
-1 & 0 & 0 & -h_{3} \\
0 & 1 & 0 & h_{2}+x_{1} \\
0 & 0 & 0 & 1
\end{array}\right] ; \quad \text { (2a) } \\
{ }^{2} \mathrm{~T}_{3}=\left[\begin{array}{cccc}
0 & 0 & -1 & 0 \\
-1 & 0 & 0 & 0 \\
0 & 1 & 0 & -h_{4}-x_{2} \\
0 & 0 & 0 & 1
\end{array}\right] \\
{ }^{6} \mathrm{~T}_{7}=\left[\begin{array}{cccc}
\cos \varphi_{2} & -\sin \varphi_{2} & 0 & h_{9} \cos \varphi_{2} \\
\sin \varphi_{2} & \cos \varphi_{2} & 0 & h_{9} \sin \varphi_{2} \\
0 & 0 & 1 & h_{10} \\
0 & 0 & 0 & 1
\end{array}\right] ;{ }^{7} \mathrm{~T}_{8}=\left[\begin{array}{llll}
0 & 0 & 1 & 0 \\
1 & 0 & 0 & 0 \\
0 & 1 & 0 & 0 \\
0 & 0 & 0 & 1
\end{array}\right](2 \mathrm{c})
\end{gathered}
$$

$$
{ }^{8} \mathrm{~T}_{9}=\left[\begin{array}{cccc}
\cos \varphi_{3} & -\sin \varphi_{3} & 0 & 0 \\
-\sin \varphi_{3} & -\cos \varphi_{3 i} & 0 & 0 \\
0 & 0 & -1 & -h_{11} \\
0 & 0 & 0 & 1
\end{array}\right]
$$

Resulting homogenous transformation matrix ${ }^{0} \mathrm{~T}_{9}$ from effector co-ordinate system to the global co-ordinate system is

${ }^{0} \mathbf{T}_{9}=\left[\begin{array}{llll}\cos \left(\varphi_{1}\right) \cdot \sin \left(\varphi_{2}\right) \cdot \cos \left(\varphi_{3}\right)-\sin \left(\varphi_{1}\right) \cdot \sin \left(\varphi_{3}\right) & -\cos \left(\varphi_{1}\right) \cdot \sin \left(\varphi_{2}\right) \cdot \sin \left(\varphi_{3}\right)-\sin \left(\varphi_{1}\right) \cdot \cos \left(\varphi_{3}\right) & \cos \left(\varphi_{1}\right) \cdot \cos \left(\varphi_{2}\right) & t_{14} \\ -\sin \left(\varphi_{1}\right) \cdot \sin \left(\varphi_{2}\right) \cdot \cos \left(\varphi_{3}\right)-\cos \left(\varphi_{1}\right) \cdot \sin \left(\varphi_{3}\right) & \sin \left(\varphi_{1}\right) \cdot \sin \left(\varphi_{2}\right) \cdot \sin \left(\varphi_{3}\right)-\cos \left(\varphi_{1}\right) \cdot \cos \left(\varphi_{3}\right) & -\sin \left(\varphi_{1}\right) \cdot \cos \left(\varphi_{2}\right) & t_{24} \\ \cos \left(\varphi_{2}\right) \cdot \cos \left(\varphi_{3}\right) & -\cos \left(\varphi_{2}\right) \cdot \sin \left(\varphi_{3}\right) & -\sin \left(\varphi_{2}\right) & t_{34} \\ 0 & 0 & 0 & 1\end{array}\right]$

where

$$
\begin{aligned}
t_{14} & =x_{2}(t)+h_{4}+\cos \left[\varphi_{1}(t)\right] \cdot\left\{h_{11} \cdot \cos \left[\varphi_{2}(t)\right]-h_{9} \cdot \cos \left[\varphi_{2}(t)\right]\right\}+\left(h_{8}+h_{10}-h_{6}\right) \cdot \sin \left[\varphi_{1}(t)\right]= \\
& =x_{2}(t)+h_{4}-\left(h_{9}-h_{11}\right) \cdot \cos \left[\varphi_{1}(t)\right] \cdot \cos \left[\varphi_{2}(t)\right]+\left(h_{8}+h_{10}-h_{6}\right) \cdot \sin \left[\varphi_{1}(t)\right] \\
t_{24} & =x_{1}(t)+h_{2}-\sin \left[\varphi_{1}(t)\right] \cdot\left\{h_{11} \cdot \cos \left[\varphi_{2}(t)\right]-h_{9} \cdot \cos \left[\varphi_{2}(t)\right]\right\}+\left(h_{8}+h_{10}-h_{6}\right) \cdot \cos \left[\varphi_{1}(t)\right]= \\
& =x_{1}(t)+h_{2}+\left(h_{9}-h_{11}\right) \cdot \sin \left[\varphi_{1}(t)\right] \cdot \cos \left[\varphi_{2}(t)\right]+\left(h_{8}+h_{10}-h_{6}\right) \cdot \cos \left[\varphi_{1}(t)\right] \\
t_{34} & =x_{3}(t)+h_{1}+h_{3}+h_{5}+h_{7}+h_{9} \cdot \sin \left[\varphi_{2}(t)\right]-h_{11} \cdot \sin \left[\varphi_{2}(t)\right]= \\
& =x_{3}(t)+h_{1}+h_{3}+h_{5}+h_{7}+\left(h_{9}-h_{11}\right) \cdot \sin \left[\varphi_{2}(t)\right]
\end{aligned}
$$

\subsection{Robot kinematic behaviour description}

For any point $\mathbf{X}$, whose coordinates in co-ordinate system $\mathbf{O}_{\mathbf{x} 9 \mathbf{y} 9 \mathbf{z} 9}$ are

$$
{ }^{9} X=\left[\begin{array}{l}
x \\
y \\
z
\end{array}\right]
$$

its global coordinates (coordinates in system $\mathbf{O}_{\mathbf{x} 0 \mathbf{y 0 z 0}}$ ) are

$$
{ }^{0} X=\left[\begin{array}{l}
x_{0} \\
y_{0} \\
z_{0}
\end{array}\right]
$$

where

$$
\begin{aligned}
x_{0} & =x_{2}(t)+h_{4}-\left(h_{9}-h_{11}\right) \cdot \cos \left[\varphi_{1}(t)\right] \cos \left[\varphi_{2}(t)\right]+\left(h_{8}+h_{10}-h_{6}\right) \cdot \sin \left[\varphi_{1}(t)\right]+ \\
& +x \cdot\left\{\cos \left[\varphi_{1}(t)\right] \cdot \sin \left[\varphi_{2}(t)\right] \cdot \cos \left[\varphi_{3}(t)\right]-\sin \left[\varphi_{1}(t)\right] \cdot \sin \left[\varphi_{3}(t)\right]\right\}+ \\
& +y \cdot\left\{-\cos \left[\varphi_{1}(t)\right] \cdot \sin \left[\varphi_{2}(t)\right] \cdot \sin \left[\varphi_{3}(t)\right]-\sin \left[\varphi_{1}(t)\right] \cdot \cos \left[\varphi_{3}(t)\right]\right\}+ \\
& +z \cdot\left\{\cos \left[\varphi_{1}(t)\right] \cdot \cos \left[\varphi_{2}(t)\right]\right\} \\
y_{0} & =x_{1}(t)+h_{2}+\left(h_{9}-h_{11}\right) \cdot \sin \left[\varphi_{1}(t)\right] \cdot \cos \left[\varphi_{2}(t)\right]+\left(h_{8}+h_{10}-h_{6}\right) \cdot \cos \left[\varphi_{1}(t)\right]+ \\
& +x \cdot\left\{-\sin \left[\varphi_{1}(t)\right] \cdot \sin \left[\varphi_{2}(t)\right] \cdot \cos \left[\varphi_{3}(t)\right]-\cos \left[\varphi_{1}(t)\right] \cdot \sin \left[\varphi_{3}(t)\right]\right\}+ \\
& +y \cdot\left\{\sin \left[\varphi_{1}(t)\right] \cdot \sin \left[\varphi_{2}(t)\right] \cdot \sin \left[\varphi_{3}(t)\right]-\cos \left[\varphi_{1}(t)\right] \cdot \cos \left[\varphi_{3}(t)\right]\right\}+ \\
& +z \cdot\left\{-\sin \left[\varphi_{1}(t)\right] \cdot \cos \left[\varphi_{2}(t)\right]\right\} \\
z_{0}= & x_{3}(t)+h_{1}+h_{3}+h_{5}+h_{7}+\left(h_{9}-h_{11}\right) \cdot \sin \left[\varphi_{2}(t)\right]+ \\
& +x \cdot\left\{\cos \left[\varphi_{2}(t)\right] \cdot \cos \left[\varphi_{3}(t)\right]\right\}+ \\
& +y \cdot\left\{-\cos \left[\varphi_{2}(t)\right] \cdot \sin \left[\varphi_{3}(t)\right]\right\}+ \\
& +z \cdot\left\{-\sin \left[\varphi_{2}(t)\right]\right\}
\end{aligned}
$$

If this point will be on axis $\mathbf{z}_{\mathbf{9}}$, then

$$
\begin{aligned}
x_{0} & =x_{2}(t)+h_{4}-\left(h_{9}-h_{11}\right) \cdot \cos \left[\varphi_{1}(t)\right] \cos \left[\varphi_{2}(t)\right]+\left(h_{8}+h_{10}-h_{6}\right) \cdot \sin \left[\varphi_{1}(t)\right]+ \\
& +z \cdot\left\{\cos \left[\varphi_{1}(t)\right] \cdot \cos \left[\varphi_{2}(t)\right]\right\} \\
y_{0} & =x_{1}(t)+h_{2}+\left(h_{9}-h_{11}\right) \cdot \sin \left[\varphi_{1}(t)\right] \cdot \cos \left[\varphi_{2}(t)\right]+\left(h_{8}+h_{10}-h_{6}\right) \cdot \cos \left[\varphi_{1}(t)\right]+ \\
& +z \cdot\left\{-\sin \left[\varphi_{1}(t)\right] \cdot \cos \left[\varphi_{2}(t)\right]\right\} \\
z_{0} & =x_{3}(t)+h_{1}+h_{3}+h_{5}+h_{7}+\left(h_{9}-h_{11}\right) \cdot \sin \left[\varphi_{2}(t)\right]+ \\
& +z \cdot\left\{-\sin \left[\varphi_{2}(t)\right]\right\}
\end{aligned}
$$

So, if we will require any position on effector rotation axis achievement in global coordinate system, it is possible, by means of these equations, to specify joints' coordinates, by which is possible to reach hereof point?

This nonlinear algebraic equations system and its solvability investigation in general case exceeds framework of presented paper [11], [12], [13].

We state only, that in general is possible to regard this structure as solvable only numerically (see below). 


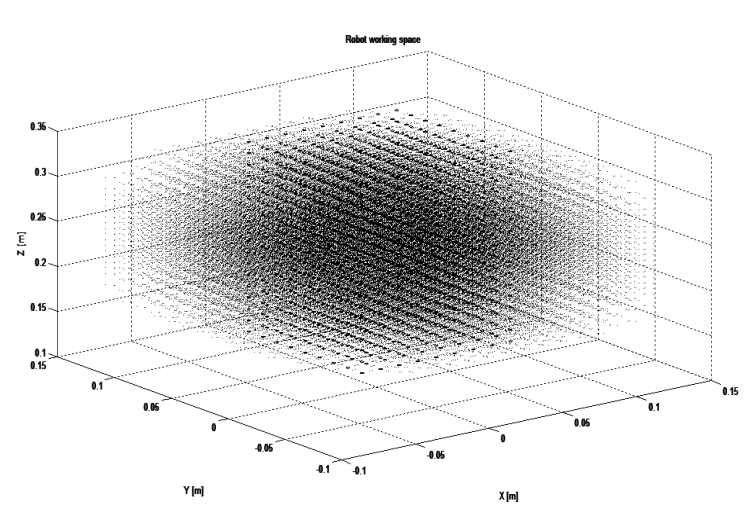

Figure 3 Calculated robot standby working place.

For any joints' coordinates possible configuration

$$
\left[\begin{array}{llllll}
x_{1} & x_{2} & x_{3} & \varphi_{1} & \varphi_{2} & \varphi_{3}
\end{array}\right]^{T}
$$

is possible by means of (3) or (4) to evaluate any effector point in global coordinates (and then determine its global velocity size and then kinetic and potential energy).

The set of all effector co-ordinate system $\mathbf{O}_{\mathbf{x} 9 \mathbf{y} 9 z 9}$ origin points for whole joints' coordinates range is substantial part of robot's standby working space and it's displayed on Figure 3.

\subsection{Inverse kinematic problem and its solution}

On the contrary, the system of equations (3) or (4), do it enables, for any required cartesian coordinates configuration of any effector point

$$
\left[\begin{array}{lll}
x_{0} & y_{0} & z_{0}
\end{array}\right]^{T}
$$

to establish such joints' coordinates

$$
\left[\begin{array}{llllll}
x_{1} & x_{2} & x_{3} & \varphi_{1} & \varphi_{2} & \varphi_{3}
\end{array}\right]^{T} \text {, }
$$

which will provide its required position in $3 \mathrm{D}$ space?

Answer to this question is answer on the question about existence and unicity of solving so-called inverse kinematic problem and its result determines required position values' dependence on time for individual joints. Real translational or angular joint position is then job of inverse dynamic problem solving, which isn't aim hereof paper.

From equations (3) is evident, that generally any required effector point absolute position

$$
\left[\begin{array}{lll}
\mathrm{x}_{0} & \mathrm{y}_{0} & \mathrm{z}_{0}
\end{array}\right]^{\mathrm{T}}
$$

faces to the 3 nonlinear algebraic equations' system for 6 unknowns and analysis its general solution and its unicity generally exceeds scope hereof paper.

We will accent only basic fact, that kinematic structure created this way presents effector body orientation inseparability from manipulator position. Thenceforth on examples we will show, that chosen trajectories of chosen effector point is possible to achieve by diverse joints control.

\subsubsection{Movement control demonstration only by means of translational joints}

We'll discover equations (4) solution for required coordinates' trajectory

$$
\left[x_{0}(t) y_{0}(t) z_{0}(t)\right]^{T}
$$

effector point, which lies on axis $\mathbf{z}_{9}$ in the distance $5.10^{-2} \mathrm{~m}$ from the origin of $\mathbf{O}_{\mathbf{x} 9 \mathbf{y} 9 \mathbf{z} 9}$. Effector body orientation is given by constant angles

$$
\varphi_{1}=-15^{\circ} ; \varphi_{2}=20^{\circ} ; \varphi_{3}=-20^{\circ}
$$

We will perform the solution only by means of translational articulated coordinates' time changes

$$
\left[x_{1}(t) x_{2}(t) x_{3}(t)\right]^{T},
$$

whereas the point with the coordinates $\left[x_{0}(t) y_{0}(t) z_{0}(t)\right]^{T}$ describes circle with radius $3,5.10^{-2} \mathrm{~m}$ around axis passing through origin $\mathbf{O}_{\mathbf{x} 9 \mathbf{y} 9 \mathbf{z}}$ parallel with axis $\mathbf{X}_{\mathbf{0}}$, in the plane parallel with global plane $\mathbf{Y}_{\mathbf{0}}, \mathbf{Z}_{\mathbf{0}}$ in the distance $5.10^{-2} \mathrm{~m}$ from the origin, with constant peripheral speed of $4,3982 \cdot 10^{-1} \mathrm{~m} / \mathrm{s}$. See Figure 4 .

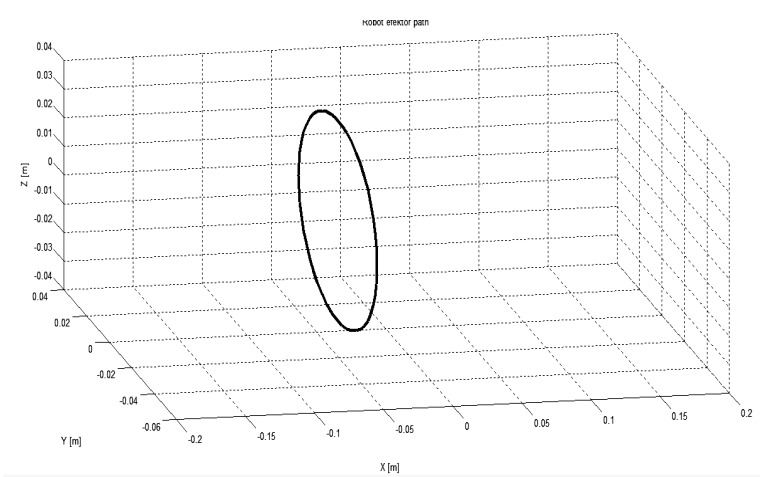

Figure 4 Required robot effector point path.

Is evident that happens an trivial situation corresponding cartesian robot type. Figure 5. shows time dependence, just as make the system (4) solution.

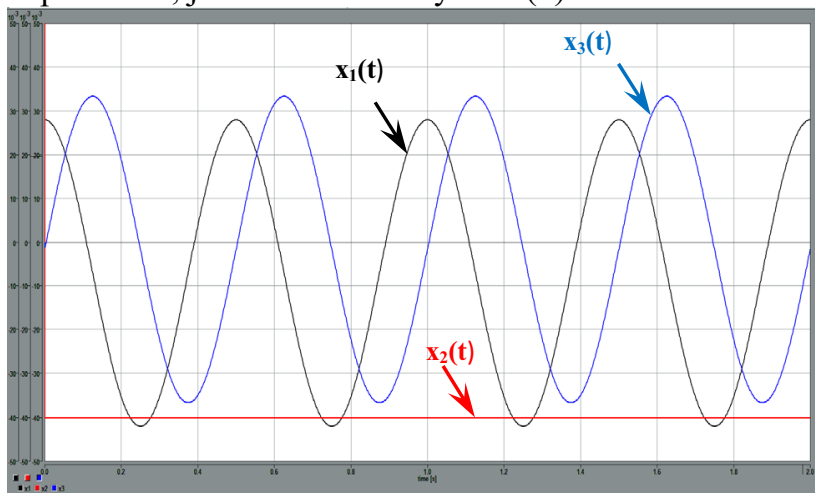

Figure $5\left[x_{1}(t) x_{2}(t) x_{3}(t)\right]^{T}$ as time functions for given parameters.

On effector orientation modification, the joints' coordinates trajectories will change, but it will conserve its form (harmonics courses), because joints' coordinates

$$
\left[x_{1}(t) x_{2}(t) x_{3}(t)\right]^{T}
$$

copy point effector trajectory. 


\subsubsection{Demonstration of movement control only by means of rotational joints}

Try to find equations (4) solution for required trajectory with coordinates

$$
\left[x_{0}(t) y_{0}(t) z_{0}(t)\right]^{T}
$$

of effector point on $\mathbf{z}_{\mathbf{9}}$ axis with fixed joins' coordinates

$$
x_{1}=0 m ; x_{2}=-2.10^{-2} m ; x_{3}=0 m
$$

We require to extended effector axis in $1 \mathrm{~m}$ distance arcs the curve from Figure 5.

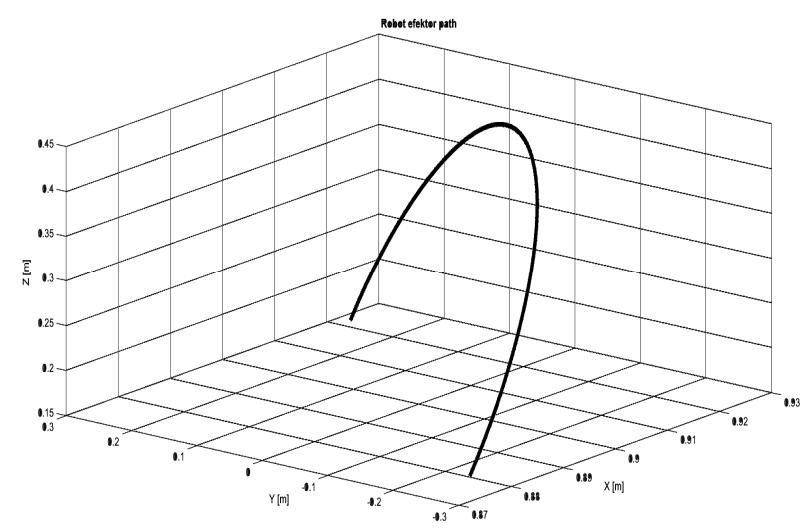

Figure 6 Required robot effector point path.

We perform the solution only by means of rotary joints, i.e. we search

$$
\varphi_{1}(t) ; \varphi_{2}(t) ; \varphi_{3}(t)
$$

satisfying our requirement. First of all we see that we can achieve this trajectory only by means of first two rotary joints. Trajectory logically not depends on third rotary joint rotation.

From third equations (4) we can solve angle $\varphi_{2}(t)$. Then second from equations (4) can be modified to form

$y_{0}-\left(x_{1}(t)+h_{2}\right)=$

$=\left\{\left(h_{9}-h_{11}-z\right) \cdot \cos \left[\varphi_{2}(t)\right]\right\} \cdot \sin \left[\varphi_{1}(t)\right]+\left(h_{8}+h_{10}-h_{6}\right) \cdot \cos \left[\varphi_{1}(t)\right]$

This equation is

$$
a \cos \varphi_{1}+b \sin \varphi_{1}=c
$$

type and it can be solve easily (multivalued). So we can obtain angle $\varphi_{1}$.

On the Figure 7 we can see the time dependence of the first two angles $\varphi_{1}, \varphi_{2}$.

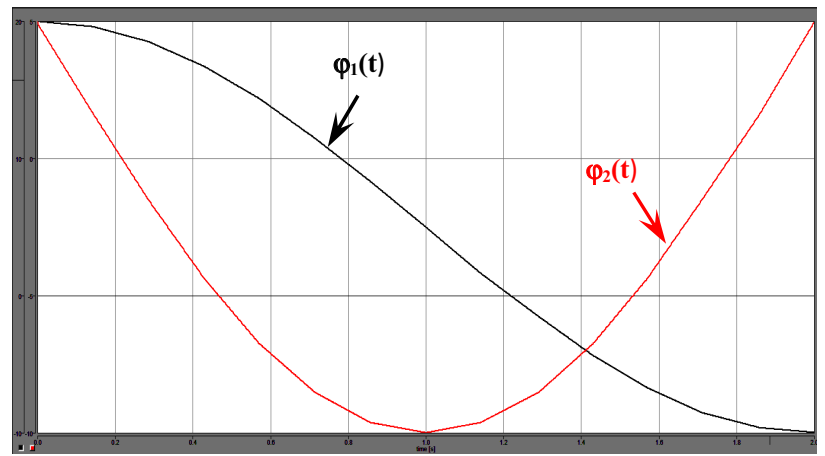

Figure 7 Rotational angles required for trajectories on Figure 6.

\section{Conclusions}

Mentioned paper is focused on measuring robot kinematic description. Robot serves to softwarecontrolled measuring effector movement in 3D space. Except basic exercise standby and available working space determination, also they are in paper described basic aspects of so-called inverse kinematic problem by means of appropriate $\mathrm{DH}$ notation and transformation matrices. I.e. required joints' coordinates finding.

Paper doesn't deal with direct and inverse dynamic problem, which concurs on kinematic problem. Motional equations derivation and control laws proposal will be subject of next parts work.

\section{References}

1. S. Crandall, D. C, .Karnopp, E.F. Kurz,. Dynamics of Mechanical and Electromechanical Systems, McGraw-Hill, NewYork (1968).

2. D. C Karnopp, R. C. Rosenberg. System dynamics. A Unifies Approach. Wiley \& Sons, (1975).

3. R. C. Rosenberg, Multiport Models in mechanics. Trans. ASME, (sept 1972)

4. Z. Úředníček, Modelling and simulation. Simulation experiment utilization at mechatronics systems' proposal, (In Czech) Inaugural dissertation. Transport and communications university ZILINA, (1997)

5. J.U. Liceaga-Castro, I. I Siller-Alcalá., J. JaimesPonce, R., Alcántara-Ramírez, and A. FerreyraRamírez, Proceedings of the 11th WSEAS International Conference on Circuits, Systems, Electronics, Control \& Signal Processing (CSECS '12). Montreux, Switzerland. December 29-31, (2012)

6. H.A. Darweesh, M. Roushdy, H.M. Ebied, and B.M. Elbagoury, Proceedings of the WSEAS International Conference Mathematical Applications in Science and Mechanics. Dubrovnik, Croatia, June 25-27, (2013)

7. S. Hubalovsky, P.Kadlec, L. Mitrovic, and P. Hanzalova, Proceedings of the 3rd International Conference on Mathematical Models for Engineering Science (MMES '12). Paris, France, December 2-4, (2012)

8. Z. Úředníček, Robotics (in Czech), T. Bata university in Zlin, Zlin (2012), ISBN 978-80-7454-223-7

9. Kohli D. and Osvatic M., ASME J. Mech. Design, (1993), 115: 922-931

10. Raghavan M. and Roth B., Kinematic analysis of the 6R manipulator of general geometry, Proc. 5th International Symposium on Robotics Research, edited by $\mathrm{H}$. Miura and S. Arimoto, MIT Press, (1990), Cambridge

11. Lee H. Y. and Reinholtz C. F., ASME J. Mech. Design, (1996), 118: 396- 404 
12. Yan Wang, Lu-bin Hang, and Ting-li Yang. Frontiers of Mechanical Engineering in China, 1:115-124, (2006). 10.1007/s11465-005-0022-7.

13. L. Bláha. Groebnerova báze a teorie ř́zení. $\mathrm{PhD}$ dissertation, Cybernetics' department, FAV, ZCU Plzen, (2011). 